\title{
Left axis deviation in patients with non-ischemic heart failure and left bundle branch block is a purely electrical phenomenon
}

Short Title: Left Axis Deviation in Left Bundle Branch Block

Authors: Saer Abu-Alrub, MD, ${ }^{* \dagger}$ Marc Strik, MD, PhD, ${ }^{* \ddagger}$ Peter Huntjens, PhD, ${ }^{\S}$ F. Daniel Ramirez, MD, MSc, ${ }^{* \ddagger}$ Mark Potse, PhD, ${ }^{\ddagger}$ Hubert Cochet, MD, PhD, ${ }^{* \ddagger}$ Hugo Marchand, MD, ${ }^{*}$ Samuel Buliard, MD, ${ }^{*}$ Romain Eschalier, MD, PhD, ${ }^{\dagger}$ Michel Haïssaguerre, $\mathrm{MD},{ }^{*}{ }^{\ddagger}$ Pierre Bordachar, MD, PhD, ${ }^{* \ddagger}$ Sylvain Ploux, MD, PhD ${ }^{* \ddagger}$

* Bordeaux University Hospital (CHU), Cardio-Thoracic Unit, 33600 Pessac-Bordeaux, France

† CHU Clermont-Ferrand, Cardiology Department, 63000 Clermont-Ferrand, France ¥ IHU Liryc, Electrophysiology and Heart Modeling Institute, fondation Bordeaux Université, F-33600 Pessac-Bordeaux, France

$\S$ Washington University in St. Louis, Division of Cardiology, 63110 St Louis MO, USA

Corresponding author: Saer Abu-Alrub (saer0330@gmail.com)

Address for correspondence:

Dr Abu-Alrub ; Service Pr Haïssaguerre, Hôpital cardiologique du Haut-Lévêque, Avenue de Magellan, 33600 Pessac, France.

\section{Conflict of interest statement:}

The authors have declared that they have no conflict of interest.

Word count: 4495 


\section{ABSTRACT}

BACKGROUND: Possible mechanisms of left axis deviation (LAD) in the setting of left bundle branch block (LBBB) include differences in cardiac electrophysiology, structure, or anatomical axis.

OBJECTIVES: We sought to clarify the mechanism(s) responsible for LAD in patients with LBBB.

METHODS: Twenty-nine patients with non-ischemic cardiomyopathies and LBBB underwent non-invasive electrocardiographic mapping (ECGi), cardiac computed tomography, and magnetic resonance imaging in order to define ventricular electrical activation, characterize cardiac structure, and determine the heart anatomical axis.

RESULTS: Sixteen patients had a normal QRS axis (NA, mean axis: $8 \pm 23^{\circ}$ ) whereas 13 patients had LAD (mean axis: $-48 \pm 13^{\circ}, p<0.001$ ). Total activation times were longer in the LAD group $(112 \pm 25$ vs $91 \pm 14 \mathrm{~ms}, p=0,01)$ due to delayed activation of the basal anterolateral region $(107 \pm 10$ vs $81 \pm 17 \mathrm{~ms}, \mathrm{p}<0.001)$. Left ventricular $(\mathrm{LV})$ activation in patients with LAD was from apex-to-base, contrasting with a circumferential pattern of activation in patients with NA. The apex-to-base delay was therefore longer in the LA group ( $95 \pm 13$ vs $64 \pm 21 \mathrm{~ms}, p<0.001$ ) and correlated with the QRS frontal axis $\left(R^{2}=0,67, p<0.001\right)$. Both groups were comparable in LV end diastolic volume (295 $\pm 84 \mathrm{vs} \mathrm{LAD:} 310 \pm 91 \mathrm{ml} ; \mathrm{p}=0.69)$, LV mass ( $177 \pm 33$ vs LAD: $180 \pm 37 g, p=0.83$ ) and anatomical axis.

CONCLUSION: Left axis deviation in left bundle branch block appears to be due to electrophysiological abnormalities rather than structural factors or the cardiac anatomical axis.

Key Words: left bundle branch block, QRS axis, left axis deviation, electrical mapping, computer modeling 


\section{INTRODUCTION}

Left bundle branch block (LBBB) is an important pathophysiologic determinant in patients with heart failure and is readily diagnosed on standard 12-lead surface ECGs. Although the alterations in ventricular depolarization produced by LBBB have been largely characterized, ${ }^{1,2}$ why certain patients with LBBB exhibit a leftward QRS axis (between $-30^{\circ}$ and $-90^{\circ}$ ) instead of a normal axis (NA, between $-30^{\circ}$ and $90^{\circ}$ ) remains unknown. Left axis deviation (LAD) could be due to differences in regional electrical activation, in left ventricular (LV) structure, or simply in the anatomical axis of the heart. A comprehensive electroanatomic evaluation of this ECG finding could clarify its mechanism and clinical significance. We therefore sought to test potential electrophysiologic, structural, and anatomic explanations for LAD in a cohort of patients with non-ischemic cardiomyopathy and LBBB undergoing cardiac resynchronization therapy (CRT) device implantation.

\section{METHODS}

This study conformed to the principles outlined in the Declaration of Helsinki on research in human subjects and was approved by the Bordeaux Centre Hospitalier Universitaire (CHU) ethics committee. All patients provided written consent to participate in the study.

\section{Study population}

Twenty-nine patients with non-ischemic dilated myocardiopathy undergoing CRT implantation were selected. All patients met the following criteria: (1) New York Heart Association (NYHA) functional class $\geq$ Il despite optimal medical therapy; (2) LV ejection fraction (LVEF) $\leq 35 \%$ during sinus rhythm; and (3) LBBB on 12-lead ECG defined as an intrinsic QRS duration of $\geq 130 \mathrm{~ms}$, broad notched or slurred $\mathrm{R}$ wave in leads $\mathrm{I}, \mathrm{aVL}, \mathrm{V} 5$, and V6, and absent $q$ waves in leads I, V5, and V6. ${ }^{3}$ A subset of the study population has been described previously in a separate study. ${ }^{4}$ LAD was defined as a QRS axis of $<-30^{\circ}$ in the frontal plane on 12-lead ECG. 


\section{Noninvasive mapping of electrical activation}

Ventricular epicardial activation maps were acquired during intrinsic activation in sinus rhythm and right ventricular apical pacing (RVAP) with an atrioventricular delay of $80 \mathrm{~ms}$ using a noninvasive, high-resolution electrocardiographic imaging (ECGi) system (ECVUE, Cardiolnsight Technologies Inc, Cleveland, $\mathrm{OH}$ ). All maps were obtained within 72 hours postCRT implantation. Briefly, body surface potentials were recorded by 252 electrodes around the entire torso. Next, a thoracic computed tomography scan was performed with the electrodes attached to the patient. The two exams were then combined and processed to reconstruct 1500 epicardial unipolar electrograms. Ventricular activation times were measured from the onset of the QRS duration (intrinsic conduction) or the pacing spike (RVAP) to the maximal negative slope of each unipolar electrogram. For comparison purposes between intrinsic and RVAP, all the subsequent calculations used the first ventricular activation as a reference. Activation times were exported to MATLAB (MathWorks, Natick, MA) to calculate mean activation times for 24 epicardial segments (basal, mid, and apical anterior, anterolateral, inferolateral, and inferior segments for both ventricles). Total activation time (TAT) was defined as the time from the earliest to the latest site of ventricular activation, excluding the first and last $5 \%$ of activation. Ventricular electrical uncoupling (VEU), a marker of interventricular dyssynchrony, was defined as the difference between mean LV and RV activation times in spontaneous rhythm. ${ }^{5}$ The apex-to-base delay was defined as the time from RV anteroapical activation to LV basal anterolateral activation in spontaneous rhythm. Finally, bullseye plots of the average electrical activation per segment in sinus rhythm and during RVAP were plotted for NA and LAD groups.

\section{Anatomic calibration}

The position of the heart within the torso was defined by its anatomical axis in three dimensions, calculated from its spatial coordinates extracted from ECGi and processed in MATLAB. Automated rotations were performed in sagittal, frontal, and transversal planes until a standardized position was achieved with the mitral valve was on top, the apex on the bottom, the right ventricular (RV) free wall on the right side, and the LV free wall on the left side. The amount of rotations required to achieve this "calibrated" position were measured and compared to examine the impact of the heart's anatomical axis on the QRS axis. 


\section{Computer simulation of the impact of altering regional activation on the surface ECG}

To explore the influence of altering electrical activation patterns while keeping the cardiac structure and position constant, we used an ECG simulator (ECGSIM v3.0.1, Nijmegen, The Netherlands). ECGSIM is an interactive computer simulation program that allows users to alter ventricular depolarization times and to study its effects on the surface ECG. ${ }^{6}$ The averaged electrical activation times per segments of each group were used as an input for the two simulations.

\section{Cardiac magnetic resonance (CMR) imaging}

CMR was used to investigate the potential influence of structural heart disease on QRS axis in a subset of patients. CMR studies were acquired using a 1.5-T system (Magnetom Avanto, Siemens Medical Systems, Erlangen, Germany) equipped with a 32-channel cardiac coil. Cine imaging was performed at a temporal resolution of 40 milliseconds. A stack of 12 short axis slices was acquired, evenly spaced over the entire LV from base to apex. Long axis slices of the LV were also acquired (four-, three-, and two-chamber views). Delayed enhanced imaging was performed 10-12 minutes after administering $0.2 \mathrm{mmol} / \mathrm{kg}$ of intravenous gadoterate meglumine. LV mass and volume were quantified on short axis slices using CMR42 software (Circle Cardiovascular Imaging Inc., Calgary, AB, Canada).

\section{CRT device implantation}

All patients underwent implantation of a CRT device. Although the RV lead was generally implanted at the RV apex, the position of the LV lead was not prespecified. The final LV pacing position was determined by coronary venous anatomy, lead stability, pacing thresholds, and assessment of phrenic nerve capture.

\section{Statistical Analysis}

Categorical variables are expressed as absolute numbers and percentages; continuous variables are expressed as mean $\pm S D$. Student $t$ or Welch tests were used to compare mean values, as appropriate. Percentages were compared by either chi square or Fisher tests. Associations between selected parameters and the QRS axis were determined using linear regression. All analyses were performed using RStudio (version 1.2.5033). P-values $<0.05$ were considered statistically significant. 


\section{RESULTS}

\section{Patient characteristics and measures of electrical dyssynchrony}

Twenty-five patients (86\%) had idiopathic dilated cardiomyopathy, $3(10 \%)$ had valvular cardiomyopathy, and 1 (3\%) had cardiomyopathy secondary to anthracycline cardiotoxicity. All 29 patients underwent noninvasive ECGi in sinus rhythm, of which 19 also had ECGi performed during RVAP and 22 also underwent CMR imaging with late gadolinium enhancement (LGE).

Thirteen patients had LBBB with LAD (mean QRS axis of $-48 \pm 13^{\circ}$ ) whereas 16 had NA (mean QRS axis of $\left.8 \pm 23^{\circ}, p<0.001\right)$. Clinical characteristics are detailed in Table 1. Patients with LAD had similar age, height, weight, LVEF, medical treatment as compared with patients with NA. Patients with LAD had a non-significant longer QRS durations ( $166 \pm 16$ vs $160 \pm 11 \mathrm{~ms}, p=0.21$ ), had longer TATs $(112 \pm 25$ vs $91 \pm 14 \mathrm{~ms}, p=0,01)$, apex-to-base time delays ( $95 \pm 13$ vs $64 \pm 21 \mathrm{~ms}$, $p<0.001)$ and VEU $(76 \pm 11 \mathrm{~ms}$ vs $65 \pm 8 m s, p=0.007)$. The distribution of these parameters is shown in Figure 1.

\section{Activation patterns during sinus rhythm}

Representative 3D electrical intrinsic activation maps of patients with LBBB and NA versus LAD are shown in Figure 2. RV activation patterns were similar for both groups, with breakthrough activation from its free wall followed by circumferential spreading toward the LV. In contrast, LV activation differed in terms of patterns and latest activated area. LBBB with NA was associated with circumferential LV activation with the latest activation seen at the mid lateral LV wall. In contrast, LBBB with LAD demonstrated the latest activation at the basal lateral LV wall, resulting in an apex-to-base activation pattern. In fact, the apex-to-base activation delay correlated significantly with $Q R S$ axis $\left(R^{2}=0.67, p<0.001\right)$, with a higher gradient associated with a more leftward axis (Figure 3). As a consequence, activation of the LV basal segments was more delayed in the LAD group: basal anterolateral ( $107 \pm 10$ vs $81 \pm 17 \mathrm{~ms}, p<0.001)$, basal inferolateral $(100 \pm 11$ vs $84 \pm 20 \mathrm{~ms}, p=0.009)$, and basal anterior ( $53 \pm 17$ vs $38 \pm 17 \mathrm{~ms}, \mathrm{p}=0.03$ ). An overview of regional electrical activation is presented in Supplementary Table 1. Bullseye plots of mean activation times are presented in Figure 4 (left panel). 


\section{Activation patterns during RVAP}

Among the 19 patients who underwent ECGi mapping during RVAP, 13 had NA (68\%) and 6 had LAD (32\%). Bullseye plots of mean activation times per group are shown in Figure 4. As expected, RV breakthrough was apical followed by RV basal activation. The site of earliest LV activation was also apical, followed by an activation front proceeding from apex-to-base. TATs were similar during sinus rhythm and RVAP in patients with NA $(95 \pm 10$ vs RVAP: $90 \pm 15 \mathrm{~ms}, p=0.28)$ and LAD ( $101 \pm 16$ vs RVAP: $101 \pm 25 \mathrm{~ms}, p=0.97)$. However, in patients with $L A D$, basal LV activation was again delayed in comparison with NA patients: basal anterolateral ( $108 \pm 16$ vs $89 \pm 17 \mathrm{~ms}, p=0.03$ ), basal anterior ( $95 \pm 29$ vs $64 \pm 28 \mathrm{~ms}, p=0.04$ ), basal inferolateral region ( $98 \pm 15$ vs $85 \pm 13, p=0.10)$. An overview of regional electrical activation is presented in Supplementary Table 2.

\section{Computer simulations}

The mean regional activation times from patients with NA were entered into ECGSIM. As shown in Figure 5, this resulted in a typical LBBB on the ECG with a NA $\left(-14^{\circ}\right)$. When the activation times of the LV base free wall were delayed in accordance with the mean regional activation times from patients with $L A D$, the QRS axis moved leftward $\left(-38^{\circ}\right)$, in keeping with clinical observations and findings on ECGi in the LAD group.

\section{Structural and anatomical differences}

Of the 22 patients who underwent CMR imaging, 13 had NA (59\%) and 9 had LAD (41\%). There were no significant differences between the two groups in terms of LVEF (NA: $22 \pm 9 \%$ vs LAD: $19 \pm 7 \%, p=0.42$ ), indexed LV end diastolic volume (NA: $154 \pm 46$ vs LAD: $171 \pm 47 \mathrm{ml} / \mathrm{m}^{2}$, $\mathrm{p}=0.69$ ), or indexed LV mass (NA: $92 \pm 20$ vs $L A D: 99 \pm 18 \mathrm{~g} / \mathrm{m}^{2}, \mathrm{p}=0.40$ ). CMR imaging results are summarized in Table 2. Myocardial fibrosis was detected in three patients (10\%), all of whom had NA ( $p=0.23)$. Among the 29 patients there were no significant differences in the heart's orientation within the torso, with similar degrees of rotation required in all planes in both NA and LAD groups to reach the standard calibrated position (sagittal: $53 \pm 11^{\circ}$ vs $53 \pm 9^{\circ}$, $p=0.90$; frontal: $-8 \pm 6^{\circ}$ vs $-11 \pm 7^{\circ}, p=0.27$; transverse: $-52 \pm 10^{\circ}$ vs $-55 \pm 11^{\circ}, p=0.51$ ) (Figure 6). 


\section{DISCUSSION}

We examined whether the QRS axis in patients with heart failure and LBBB is determined by electrophysiologic, structural, or anatomic characteristics. The results of our study support a purely electrophysiologic explanation for LAD in patients with LBBB, with no discernible associations of structural characteristics (cardiac size, LV dimensions, or LV mass) or cardiac anatomical axis with the QRS axis.

\section{LAD is an electrical phenomenon}

LV activation in the setting of LBBB has been widely described in the literature as being circumferential, spreading from the RV free wall to the LV lateral wall. ${ }^{5,7}$ However, important differences in activation exist among patients with LBBB based on their QRS axis. Patients with LBBB plus LAD exhibit an apex-to-base activation sequence with a marked delay in activation of the LV base. This added delay deviates electrical forces, resulting in a leftward QRS axis - an activation sequence that can be mimicked by RVAP. ${ }^{8}$ This explanation is bolstered by the strong correlation between the apex-to-base gradient and QRS axis, with a higher gradient being associated with a more leftward axis. The ECGSIM simulations further support an electrophysiologic etiology for LAD as anatomical and structural factors were not altered in those models.

Our results are in line with body surface mapping data from patients with LBBB and NA or LAD published by Sohi et al. ${ }^{9}$ In their study, the electrical force produced by LV activation remained anterior throughout depolarization in patients with NA, but moved toward the left shoulder and left upper back in patients with LAD. Based on this observation, the authors speculated that in LBBB with LAD a further selective slowing of depolarization existed anterobasally with the terminal positive potentials directed posterosuperiorly. Our data from advanced mapping technologies support this hypothesis, while also accounting for the heart's position in the torso via computed tomography and cardiac structural factors via CMR.

\section{Mechanisms of LAD in LBBB}

Several hypotheses have been proposed to explain mechanisms of LAD in patients with LBBB. In 1960, Rosenbaum classified LBBB into predivisional and divisional types. ${ }^{10}$ 
Predivisional LBBB referred to a conduction block in the main stem, while divisional LBBB referred to an interruption within divisional branches. Many authors postulated that LBBB with LAD was the result of a two-level block: a divisional left anterior hemiblock in addition to a predivisional LBBB. ${ }^{11-13}$ Cannom et al were the first to provide empirical support for this theory by demonstrating a leftward shift in mean QRS axis following an atrial extrastimulus in six patients with LBBB. ${ }^{13}$ Several years later, Gressard similarly used an electrophysiologic approach to explain axis differences in patients with LBBB. He observed the same response to atrial extrastimulus and concluded that LBBB with NA was due to slowed but persistent conduction in both anterior and posterior rami of the left bundle. In contrast, in LBBB with LAD, conduction was blocked in the anterior ramus. ${ }^{12}$ However, in the study by Cannom and al, the QRS axis deviation was relatively modest, not deviating past $-30^{\circ}$ except in one patient. Moreover, Rosenbaum et al found that when a left anterior hemiblock is produced experimentally, further sectioning of the trunk of the branch results in disappearance of the marked QRS LAD. ${ }^{14}$ Furthermore, ECGs of patients with intermittent LBBB have shown that the QRS axis with LBBB often does not correspond to the QRS axis without LBBB, therefore underlying left anterior hemiblock would not account for LAD during LBBB in most patients. ${ }^{15-17}$ Finally, Riedlbauchová et al recently performed endocardial electroanatomic mapping in 11 patients with LBBB and did not observe early activation of the left anterior or posterior fascicles regions in any patient. Instead, the LV was consistently activated through transmyocardial conduction. ${ }^{18}$

Our mapping study does not support the hypothesis of residual anterograde Purkinje conduction as the etiology for LAD in patients with LBBB. In patients with NA, activation during spontaneous rhythm and during RVAP differed in terms of pattern but not duration. During RVAP ventricular activation is not dependent on the Purkinje network, but depends entirely on transmyocardial conduction. Thus, if the Purkinje network was involved in LV activation during sinus rhythm in patients with LBBB and NA, LV activation times would be expected to be shorter in sinus rhythm than during RVAP. Moreover, the persistently delayed activation of the basal anterior and basal lateral regions of the LV in patients with LBBB and LAD during RVAP favors the existence of additional conduction disturbances in these patients. Thus, LAD in patients with LBBB appears to be due to additional 
transmyocardial conduction disturbances that result in delayed activation of the basal anterior and basal lateral regions of the LV.

Another proposed theory was that LV enlargement was the driver of QRS axis deviation in patients with LBBB. ${ }^{15,19}$ Our results do not support this explanation as we found no relation between the QRS axis and cardiac structural characteristics (cardiac size, LV dilatation, or LV wall mass) or the anatomical axis of the heart. Our findings align well with a prospective study by Das et al that did not find a correlation between the QRS axis and LVEF in 300 consecutive patients with LBBB. ${ }^{20}$ Although dilated cardiomyopathy is commonly found in patients with LBBB and LAD, LV dilatation alone does not seem to adequately account for the LAD. However, it is possible that LAD is a marker of more advanced electrophysiological remodeling. Our findings may prove useful to interpret ongoing investigations of LV septal pacing and conduction system pacing. ${ }^{21}$ These evolving technologies rely on underlying electrophysiological substrates in the LV which may thus vary between LBBB patients.

\section{Characteristics of the signal}

When using ECGi to investigate axial differences in the frontal plane, it is important to consider potential differences between endocardial and epicardial cardiac layers. As compared to frontal bipolar leads, unipolar chest electrodes used for the surface ECG and ECGi are subjected to a larger contribution of epicardial signals, as they are in closer proximity with the epicardium. However, the recorded signal is also composed by transmembrane potential differences in intramural and endocardial cardiac layers and therefore rather reflects a net activation of the cardiac wall. ${ }^{22}$ The extent to which noncontact mapping represents local activation has been investigated by Thiagalingam et al in murine ventricles. They compared a non-contact mapping system (EnSite) with a grid of 5060 plunge needles, each holding four contact electrodes recording from the endocardium, two intramural sites, and the epicardium. Noncontact electrograms correlated equally well in morphology and timing across all layers confirming that noncontact electrograms are a composed signal rather than a local measurement. ${ }^{23}$ On the other hand, while the abnormalities which cause LBBB originate in the endocardium, it is the net activation of all cardiomyocytes which give rise to the frontal plane axis. While the agreements between ECGi and frontal axis ECG are not perfect, the non-invasive single-beat ECGi mapping system 
remains the tool of choice for investigating the etiology of ECG axis deviation in LBBB patients.

\section{Study limitations}

Previous validation studies of ECGi have shown that agreement of non-invasive electrocardiographic mapping and contact mapping is limited. However, it is important to note that the agreement was poor for narrow QRS patterns but that there was good correlation for wide QRS patterns. ${ }^{24}$ Moreover, our results were highly consistent for the two groups. Besides, our study only evaluated patients with reduced ejection fraction. Whether our findings hold true in patients with LBBB and preserved ejection fraction remains to be investigated.

\section{CONCLUSION}

LAD in patients with heart failure and LBBB is due to additional transmyocardial conduction disturbances that result in markedly delayed activation of the basal anterolateral regions of the LV wall, and are not related to the anatomical axis of the heart or to cardiac structural factors.

\section{Funding:}

This work received financial support from the French Government as part of the "Investments of the Future" program managed by the National Research Agency (ANR), Grant reference ANR-10-IAHU-04. FDR is supported by a Canadian Institutes of Health Research Banting Postdoctoral Fellowship. 


\section{References}

1. Vassallo JA, Cassidy DM, Marchlinski FE, et al.: Endocardial activation of left bundle branch block. Circulation 1984; 69:914-923.

2. Strik M, Regoli F, Auricchio A, Prinzen F: Electrical and Mechanical Ventricular Activation During Left Bundle Branch Block and Resynchronization. J Cardiovasc Transl Res 2012; 5:117-126.

3. Surawicz B, Childers R, Deal BJ, Gettes LS: AHA/ACCF/HRS Recommendations for the Standardization and Interpretation of the Electrocardiogram: Part III: Intraventricular Conduction Disturbances: A Scientific Statement From the American Heart Association Electrocardiography and Arrhythmias Committee, Council on Clinical Cardiology; the American College of Cardiology Foundation; and the Heart Rhythm Society: Endorsed by the International Society for Computerized Electrocardiology. Circulation [Internet] 2009 [cited 2020 Apr 15]; 119. Available from: https://www.ahajournals.org/doi/10.1161/CIRCULATIONAHA.108.191095

4. Strik M, Ploux S, Huntjens PR, et al.: Response to cardiac resynchronization therapy is determined by intrinsic electrical substrate rather than by its modification. Int J Cardiol 2018; 270:143-148.

5. Ploux S, Lumens J, Whinnett Z, et al.: Noninvasive Electrocardiographic Mapping to Improve Patient Selection for Cardiac Resynchronization Therapy: Beyond QRS Duration and Left Bundle Branch Block Morphology. J Am Coll Cardiol 2013; 61:2435-2443.

6. van Oosterom A, Oostendorp TF: ECGSIM: an interactive tool for studying the genesis of QRST waveforms. Heart 2004; 90:165-168.

7. Wyndham CR, Meeran MK, Smith T, et al.: Epicardial activation of the intact human heart without conduction defect. Circulation 1979; 59:161-168.

8. Eschalier R, Ploux S, Lumens J, et al.: Detailed analysis of ventricular activation sequences during right ventricular apical pacing and left bundle branch block and the potential implications for cardiac resynchronization therapy. Heart Rhythm Elsevier, 2015; 12:137-143.

9. Sohi GS, Flowers NC, Horan LG, Sridharan MR, Johnson JC: Comparison of total body surface map depolarization patterns of left bundle branch block and normal axis with left bundle branch block and left-axis deviation. Circulation 1983; 67:660-664.

10. Rosenbaum MB: Types of left bundle branch block and their clinical significance. $\mathrm{J}$ Electrocardiol 1969; 2:197-206.

11. Lichstein E, Mahapatra R, Gupta PK, Chadda KD: Significance of complete left bundle branch block with left axis deviation. Am J Cardiol 1979; 44:239-242.

12. Gressard A: Left bundle branch block with left-axis deviation: an electrophysiologic approach. Am J Cardiol 1983; 52:1013-1016. 
13. Cannom DS, Goldreyer BN, Damato AN: Atrioventricular Conduction System in Left Bundle-Branch Block with Normal QRS Axis. Circulation 1972; 46:129-137.

14. Rosenbaum MB: The hemiblocks: diagnostic criteria and clinical significance. Mod Concepts Cardiovasc Dis 1970; 39:141-146.

15. Blondeau M: Complete left bundle branch block with marked left axis deviation of qrs clinical and anatomical study. Adv Cardiol 1975; 14:25-38.

16. DePasquale NP, Bruno MS: Left bundle branch block and abnormal left axis deviation without left anterior hemiblock. Am J Cardiol Elsevier, 1973; 32:98-100.

17. Swiryn S, Abben R, Denes P, Rosen KM: Electrocardiographic determinants of axis during left bundle branch block: Study in patients with intermittent left bundle branch block. Am J Cardiol 1980; 46:53-58.

18. Riedlbauchová L, Adla T, Suchánek V, et al.: Is left bundle branch block pattern on the ECG caused by variable ventricular activation sequence? Pacing Clin Electrophysiol 2020; 43:486-494.

19. Dhingra RC, Amat-Y-Leon F, Wyndham C, et al.: Significance of left axis deviation in patients with chronic left bundle branch block. Am J Cardiol Elsevier, 1978; 42:551556.

20. Das MK, Cheriparambil K, Bedi A, et al.: Prolonged QRS duration (QRS $>/=170 \mathrm{~ms}$ ) and left axis deviation in the presence of left bundle branch block: A marker of poor left ventricular systolic function? Am Heart J 2001; 142:756-759.

21. Arnold AD, Whinnett ZI, Vijayaraman P: His-Purkinje Conduction System Pacing: State of the Art in 2020. Arrhythmia Electrophysiol Rev 2020; 9:136-145.

22. Kligfield P, Gettes LS, Bailey JJ, et al.: Recommendations for the Standardization and Interpretation of the Electrocardiogram: Part I: The Electrocardiogram and Its Technology: A Scientific Statement From the American Heart Association Electrocardiography and Arrhythmias Committee, Council on Clinical Cardiology; the American College of Cardiology Foundation; and the Heart Rhythm Society Endorsed by the International Society for Computerized Electrocardiology. Circulation 2007; 115:1306-1324.

23. Thiagalingam A, Wallace EM, Boyd AC, et al.: Noncontact mapping of the left ventricle: insights from validation with transmural contact mapping. Pacing Clin Electrophysiol PACE 2004; 27:570-578.

24. Duchateau J, Sacher F, Pambrun T, et al.: Performance and limitations of noninvasive cardiac activation mapping. Heart Rhythm 2019; 16:435-442. 
Table 1. Patients clinical characteristics

\begin{tabular}{|c|c|c|c|}
\hline Variables & Normal Axis $(n=16)$ & Left Axis ( $n=13)$ & p Value \\
\hline Age, years & $64 \pm 11$ & $68 \pm 11$ & 0.40 \\
\hline Male & $11(70)$ & $7(54)$ & 0.57 \\
\hline Height, cm & $168 \pm 9$ & $166 \pm 9$ & 0.58 \\
\hline Weight, kg & $80 \pm 19$ & $69 \pm 12$ & 0.07 \\
\hline Ejection fraction, \% & $30 \pm 7$ & $29 \pm 5$ & 0.79 \\
\hline Paroxysmal atrial fibrillation & $2(11)$ & $2(16)$ & 1 \\
\hline $\begin{array}{l}\text { Glomerular filtration rate, } \\
\left(\mathrm{ml} / \mathrm{min} / 1,73 \mathrm{~m}^{2}\right)\end{array}$ & $73 \pm 20$ & $70 \pm 22$ & 0.68 \\
\hline \multicolumn{4}{|l|}{ Symptoms, N (\%): } \\
\hline - NYHA 2 & $11(70)$ & $6(46)$ & 0.39 \\
\hline - NYHA 3 & $3(19)$ & $7(54)$ & 0.06 \\
\hline - NYHA 4 & $2(11)$ & $0(0)$ & 0.49 \\
\hline \multicolumn{4}{|l|}{ Cardiovascular risk factor: } \\
\hline - Hypertension & $7(43)$ & $2(16)$ & 0.12 \\
\hline - Diabetes & $6(38)$ & $1(8)$ & 0.09 \\
\hline - Dyslipidemia & $9(56)$ & $3(24)$ & 0.13 \\
\hline \multicolumn{4}{|l|}{ Treatments: } \\
\hline - ACE/ARB & $16(100)$ & $12(92)$ & 0.44 \\
\hline - Beta blockers & $11(70)$ & $10(77)$ & 0.69 \\
\hline - MRA & $11(70)$ & $8(61)$ & 0.71 \\
\hline - Amiodarone & $2(11)$ & $3(23)$ & 0.63 \\
\hline - $\quad$ Furosemide, mg & $26 \pm 28$ & $47 \pm 73$ & 0.34 \\
\hline
\end{tabular}


Table 2. Baseline left ventricular characteristics on magnetic resonance imaging

\begin{tabular}{|c|c|c|c|}
\hline Variables & Normal Axis $(n=13)$ & Left Axis Deviation ( $n=9$ ) & p Value \\
\hline Ejection fraction, \% & $22 \pm 9$ & $19 \pm 7$ & 0.42 \\
\hline End diastolic volume, $\mathrm{ml}$ & $295 \pm 84$ & $310 \pm 91$ & 0.69 \\
\hline $\begin{array}{l}\text { Indexed end diastolic volume, } \\
\mathrm{ml} / \mathrm{m}^{2}\end{array}$ & $154 \pm 46$ & $171 \pm 47$ & 0.41 \\
\hline Myocardial mass, g & $177 \pm 33$ & $180 \pm 37$ & 0.83 \\
\hline Indexed myocardial mass, $\mathrm{g} / \mathrm{m}^{2}$ & $92 \pm 20$ & $99 \pm 18$ & 0.40 \\
\hline
\end{tabular}


Abu-Alrub et al: Left axis deviation in left bundle branch block
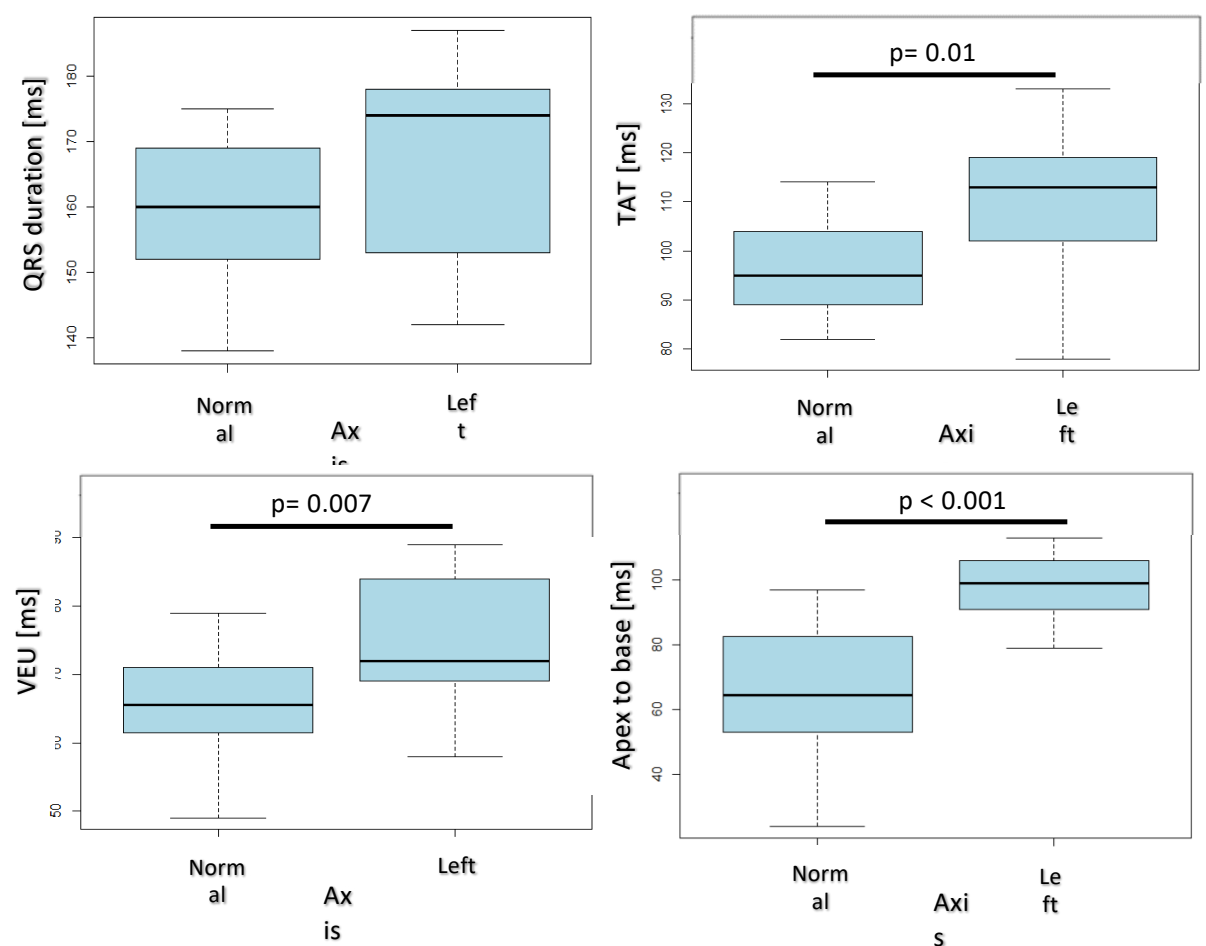

Figure 1. Distribution of QRS duration, TAT, VEU, and apex-to-base delay stratified by QRS axis. Blue box represents $50 \%$ of the distribution of the values, the black line is the median, dashed lines each represent $25 \%$ distribution of the values. TAT: total activation time; VEU: ventricular electrical uncoupling. 


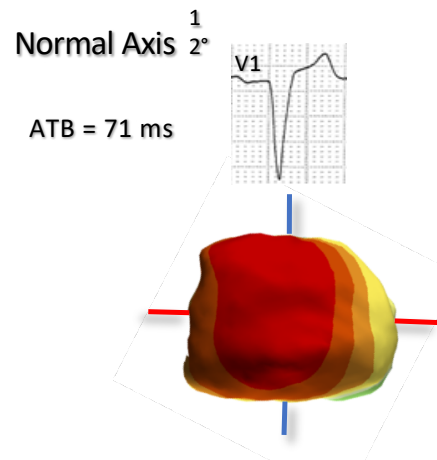

AP

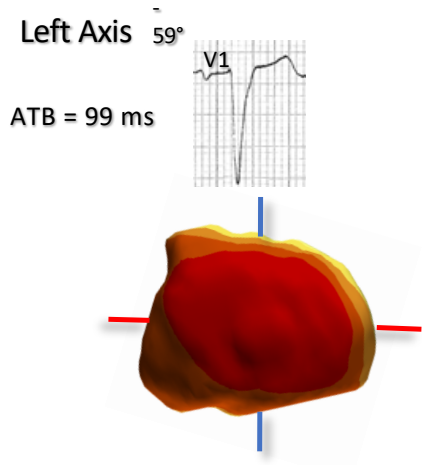

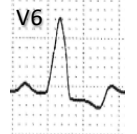



LAO

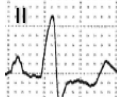

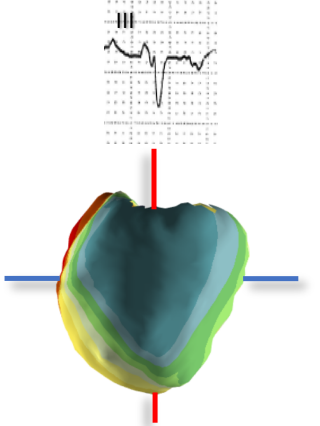

LL

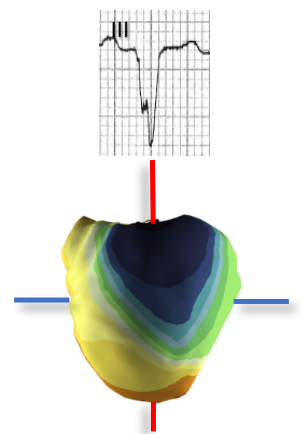

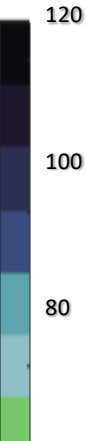

60

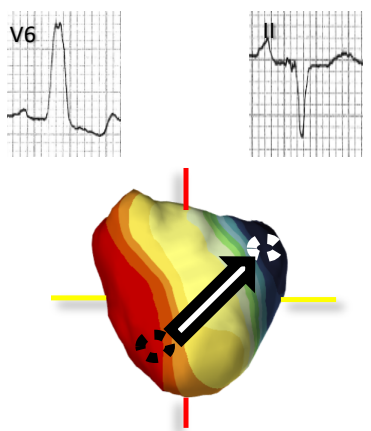

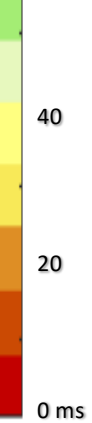

Figure 2. 3D activation maps of a typical normal axis $\angle B B B$ (top panel) and a left axis $\angle B B B$ (bottom panel). The dashed circles represent right ventricular anteroapical region (black) and left ventricular basal anterolateral region (white). The arrows depict apex-to-base (ATB) gradients. LBBB: left bundle branch block. 
Abu-Alrub et al: Left axis deviation in left bundle branch block

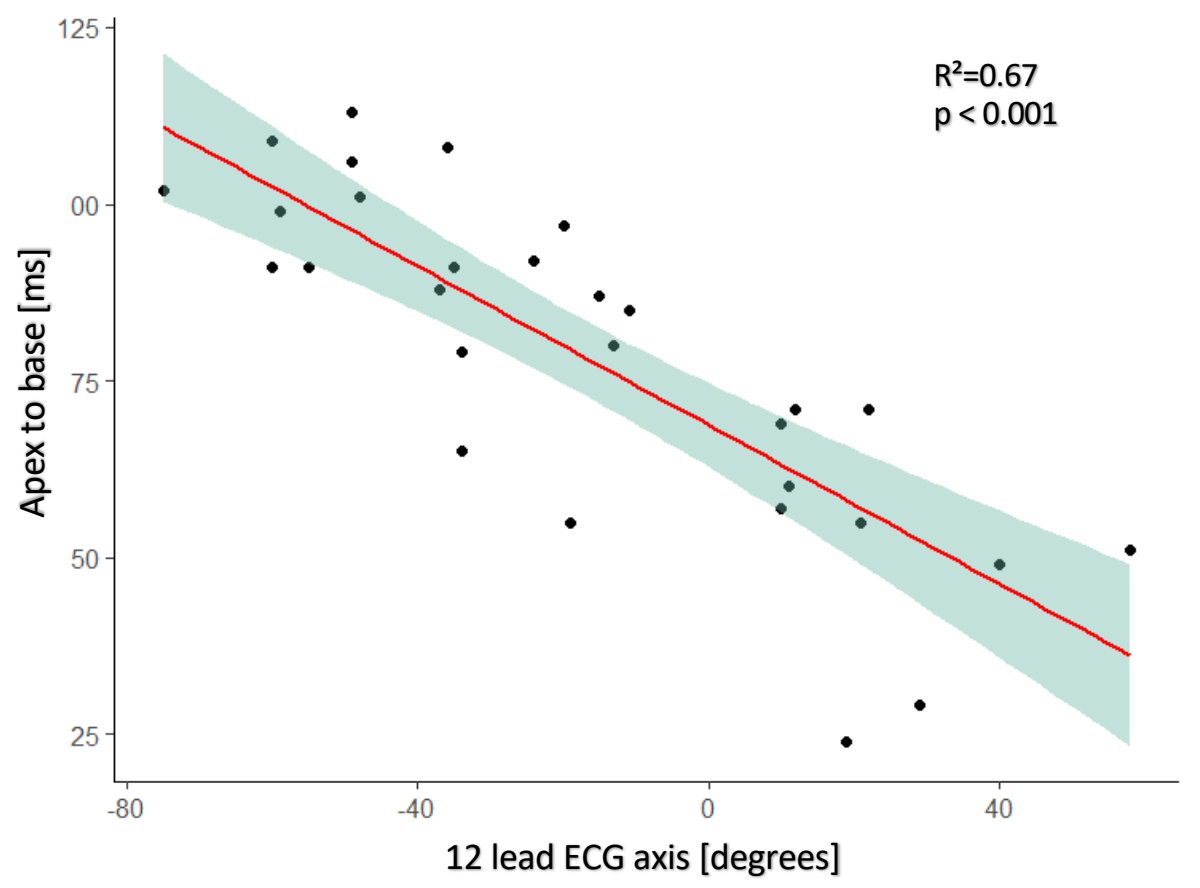

Figure 3. Correlation of apex-to-base gradient with QRS axis on 12-lead ECG. Linear trend is plotted with its $95 \%$ confidence interval. 
Abu-Alrub et al: Left axis deviation in left bundle branch block

Sinus Rhythm

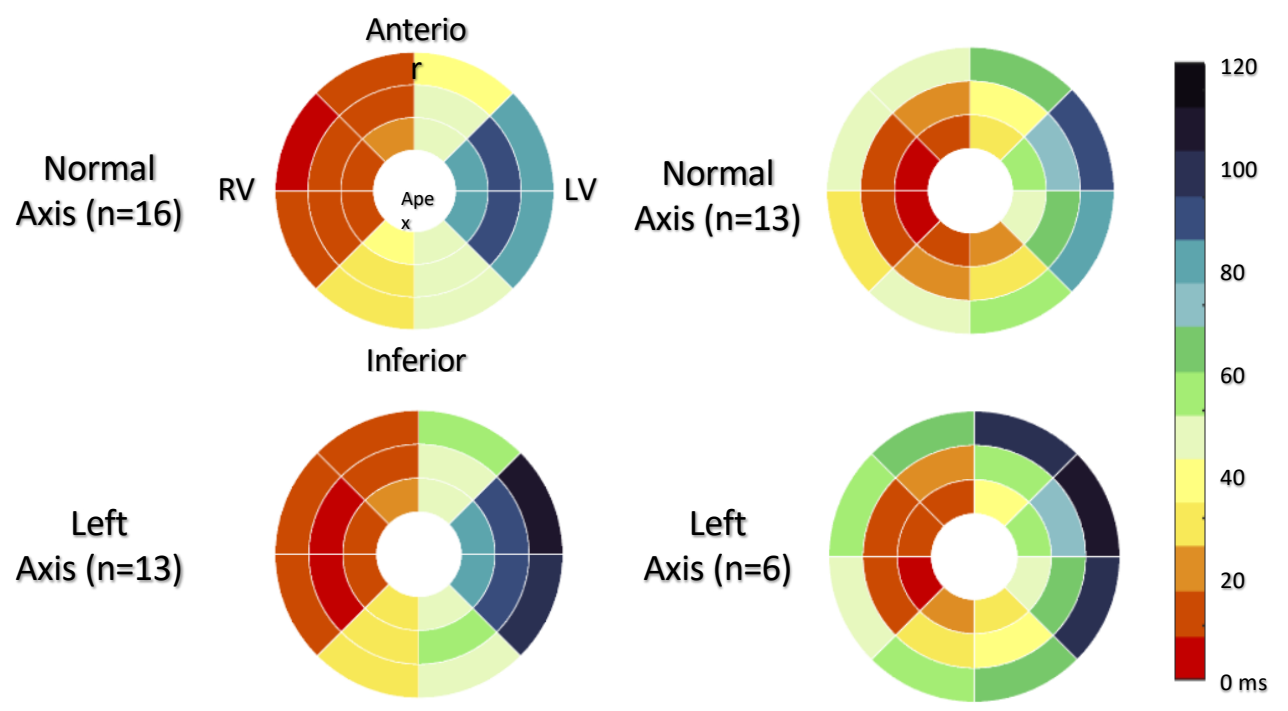

Figure 4: Ventricular activation times of all normal axis (top) and leftward axis deviation (bottom) averaged per segment during sinus rhythm (left) and right ventricular apical pacing (RVAP, right). 


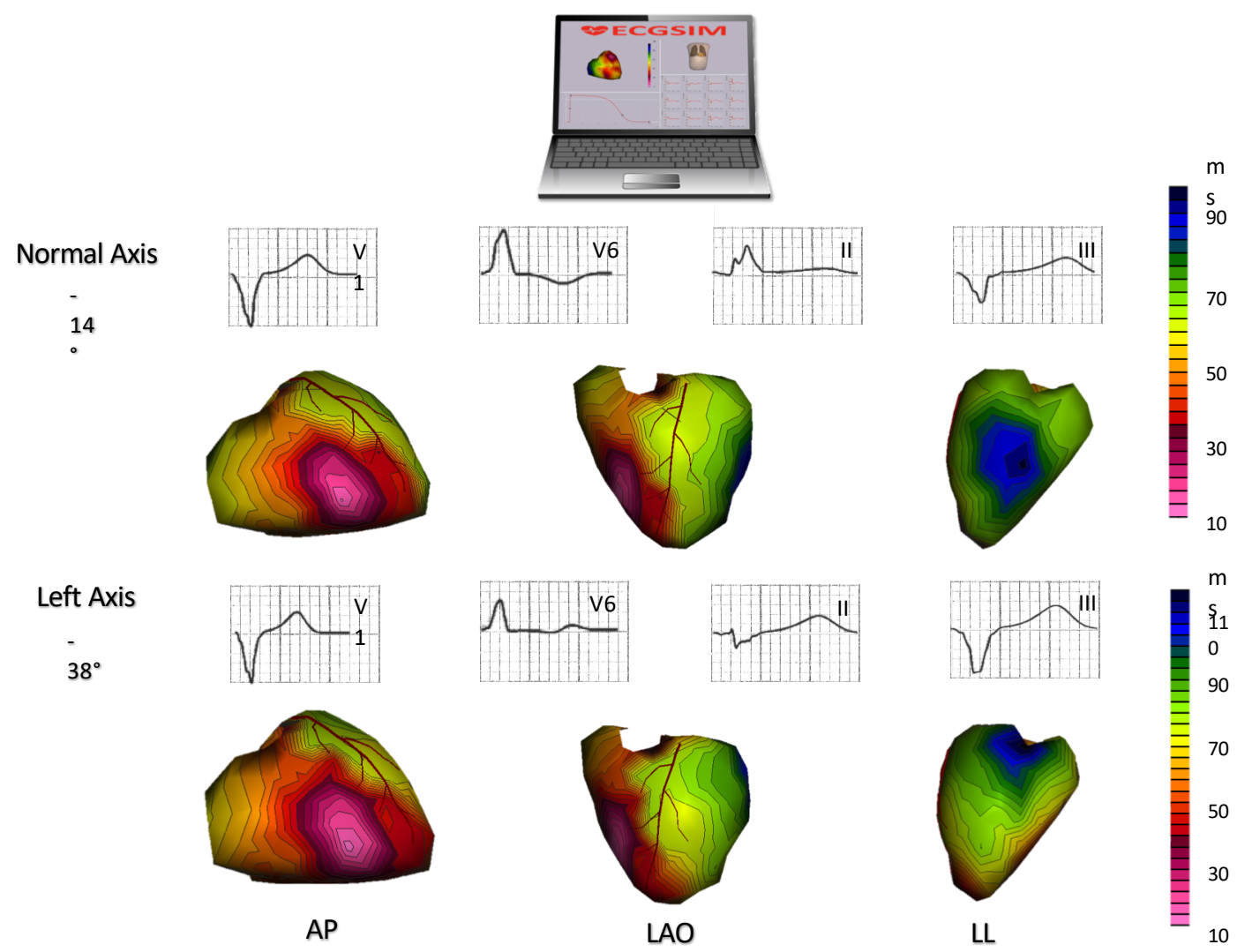

Figure 5. Simulated epicardial activation map of normal axis $\angle B B B$ (top panel) and left axis $\angle B B B$ (bottom panel). $L B B B=$ left bundle branch block. 
Abu-Alrub et al: Left axis deviation in left bundle branch block

Baseline Position

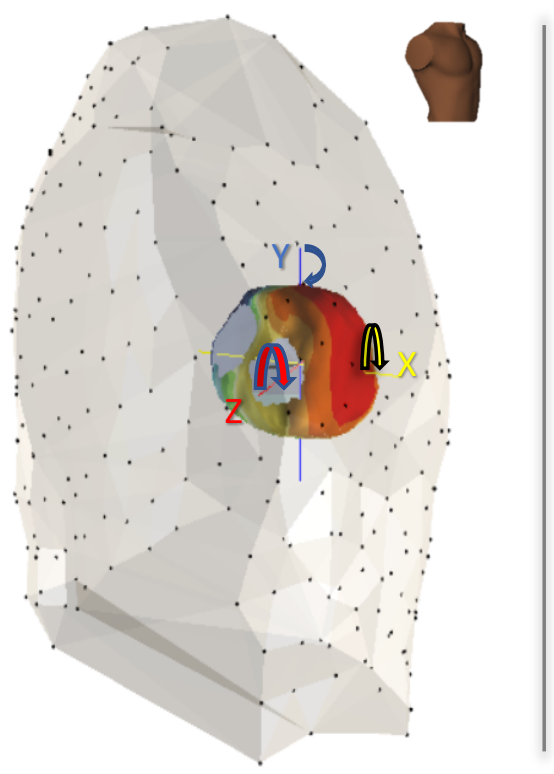

Calibrated Position

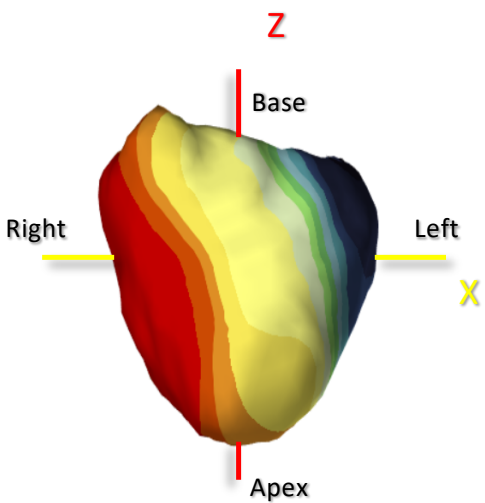

Figure 6. Representation of baseline and calibrated position of the heart within the torso. Left panel: Baseline anatomical position of the heart in the torso. Each point represents one of the 252 body surface electrodes. Direction of rotation for each plane is shown. Right panel: The heart in calibrated (standard) position after rotations in sagittal $(X)$, frontal $(Y)$ and transverse (Z) planes. 